This item was submitted to Loughborough's Research Repository by the author.

Items in Figshare are protected by copyright, with all rights reserved, unless otherwise indicated.

\title{
Dynamics of benzene excimer formation from the parallel-displaced dimer
}

PLEASE CITE THE PUBLISHED VERSION

https://doi.org/10.1039/c8cp06354k

\section{PUBLISHER}

(C) Royal Society of Chemistry (RSC)

\section{VERSION}

AM (Accepted Manuscript)

\section{PUBLISHER STATEMENT}

This paper was accepted for publication in the journal Physical Chemistry Chemical Physics and the definitive published version is available at https://doi.org/10.1039/c8cp06354k

\section{LICENCE}

CC BY-NC-ND 4.0

\section{REPOSITORY RECORD}

Cardozo, Thiago M., Andre P. Galliez, Itamar Borges, Felix Plasser, Adelia J. Aquino, Mario Barbatti, and Hans Lischka. 2019. "Dynamics of Benzene Excimer Formation from the Parallel-displaced Dimer". figshare. https://hdl.handle.net/2134/36520. 


\title{
Dynamics of Benzene Excimer Formation from the Parallel-Displaced Dimer
}

\author{
Thiago Messias Cardozo, ${ }^{1, *}$ Andre Pessoa Galliez, ${ }^{1}$ Itamar Borges Jr., ${ }^{2}$ Felix Plasser, ${ }^{3}$ \\ Adelia J. A. Aquino, ${ }^{4,5,6}$ Mario Barbatti, ${ }^{7,}$ Hans Lischka, ${ }^{4,5,6,{ }^{*}}$ \\ ${ }^{l}$ Instituto de Química, Universidade Federal do Rio de Janeiro, \\ Rio de Janeiro, RJ 21941-909, Brazil. \\ ${ }^{2}$ Departamento de Química, Instituto Militar de Engenharia, \\ Praça General Tibúrcio, 80, 22290-270 Rio de Janeiro, Brazil \\ ${ }^{3}$ Department of Chemistry, Loughborough University, LE11 3TU, United Kingdom \\ ${ }^{4}$ School of Pharmaceutical Sciences and Technology, Tianjin University, \\ Tianjin 300072, P.R. China \\ ${ }^{5}$ Department of Chemistry and Biochemistry, Texas Tech University, \\ Lubbock, Texas 79409, United States \\ ${ }^{6}$ Institute of Theoretical Chemistry, University of Vienna, \\ Währingerstraße 17, 1090 Vienna, Austria \\ ${ }^{7}$ Aix Marseille Univ, CNRS, ICR, Marseille, France. \\ *Corresponding authors.E-mail addresses:TMC: thiago@iq.ufrj.br; MB: \\ mario.barbatti@univ-amu.fr; HL: hans.lischka@univie.ac.at
}




\begin{abstract}
Excimers play a key role in a variety of excited-state processes, such as exciton trapping, fluorescence quenching, and singlet-fission. The dynamics of benzene excimer formation in the first 2 ps after $S_{1}$ excitation from the parallel-displaced geometry of the benzene dimer is reported here. It was simulated via nonadiabatic surface-hopping dynamics using the second-order algebraic diagrammatic construction $(\operatorname{ADC}(2))$. After excitation, the benzene rings take $\sim 0.5-1.0 \mathrm{ps}$ to approach each other in a parallel-stacked structure of the $\mathrm{S}_{1}$ minimum and stay in the excimer region for $\sim 0.1-0.4 \mathrm{ps}$ before leaving due to excess vibrational energy. The $\mathrm{S}_{1}-\mathrm{S}_{2}$ gap widens considerably while the rings visit the excimer region in the potential energy surface. Our work provides detailed insight into correlations between nuclear and electronic structure in the excimer and shows that decreased ring distance goes along with enhanced charge transfer and that fast exciton transfer happens between the rings, leading to the equal probability of finding the exciton in each ring after around 1.0 ps.
\end{abstract}




\section{TOC Figure}

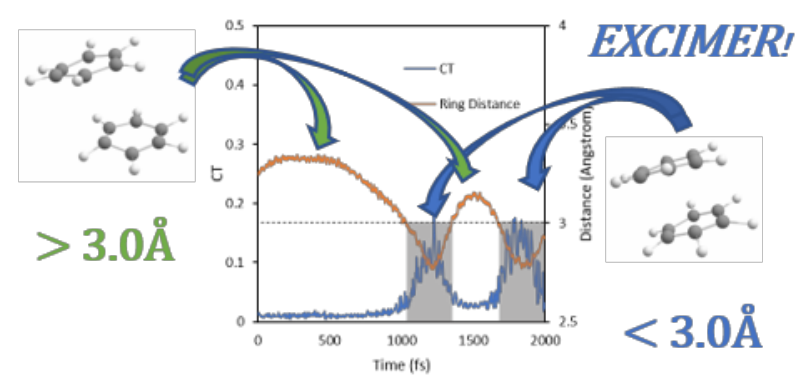

Charge transfer, exciton localization and time scales in benzene excimer formation after a $\mathrm{S}_{0}-\mathrm{S}_{1}$ transition from the parallel-displaced structure were characterized by surfacehopping dynamics. 


\section{Introduction}

Excimers are bound excited dimers without a bound ground state. ${ }^{1,2}$ Excimers and exciplexes (a heterodimer excimer) are important excited-state species involved in a number of photochemical and photophysical processes. They play crucial roles in optoelectronic properties of organic materials, underlying diverse phenomena such as fluorescence quenching ${ }^{3}$ and singlet-fission ${ }^{4-7}$ in aggregated systems, photorelaxation of nucleic acids, ${ }^{8,9}$ and charge/energy diffusion in organic photovoltaic materials. ${ }^{10-12}$ The excimer bonding is usually rationalized as originating from two types of interactions, namely, exciton resonance and charge resonance. ${ }^{2}$ The benzene excimer-the focus of the present work - is usually considered to have some degree of charge resonance interaction, but the precise proportion of the contributions is not yet established. ${ }^{13-15}$

The benzene dimer (Figure 1) is a prototypical system for the study of excimers, for which there is an abundance of both theoretical and experimental data. ${ }^{16-40}$ The two lowest-energy minima in the $S_{0}$ potential energy surface (PES) of the benzene dimer were previously established as a T-shaped asymmetric top and a parallel-displaced geometry. High-level calculations based on basis set extrapolation and explicitly correlated (F12) methods ${ }^{41}$ show that the T-shaped and parallel displaced structures are almost isoenergetic with the former structure slightly more stable $28,36-40$. It has been shown, however, that the T-shaped structure is only a saddle point and that the a tilted T-shaped structure is the minimum ${ }^{34,36}$.

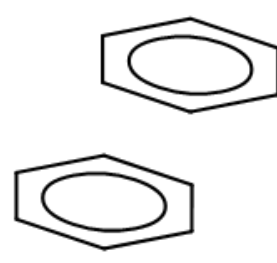

(A)

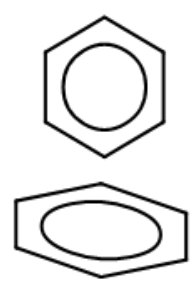

(B)

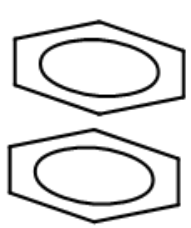

(C)

Figure 1. Parallel-displaced (A), T-shaped (B), and parallel-stacked (C) configurations of the benzene dimer.

The precise nature of the mechanism of formation of the $\mathrm{S}_{1}$ benzene excimer is also a matter of debate. It is generally agreed based on both theoretical and experimental 
data that the $\mathrm{S}_{1}$ excimer has a parallel-stacked structure. ${ }^{14,18,42,43}$ Direct formation of the excimer from the $S_{0} \rightarrow S_{1}$ excitation has been contested by Shinohara and Nishi based on the lifetime of the resulting dimer and the lack of broadening in the fluorescence excitation spectrum. ${ }^{22}$ The same authors proposed that a barrier in $S_{1}$ between the Tshaped complex and the parallel-stacked structure prohibits the formation of the excimer via this path, and rather, that the excimer is formed via the $S_{0} \rightarrow S_{2}$ transition, followed by internal conversion to $\mathrm{S}_{1}{ }^{22}$ These results have been questioned by the evidence that the $S_{2}$ undergoes ultrafast internal conversion $(\sim 40 \mathrm{fs})$ to $S_{1}{ }^{44}$ Other works propose that the $S_{0} \rightarrow S_{1}$ excitation of the T-shaped structure can lead to the excimer via tunneling. ${ }^{14}$ More recently, it has been argued that resonance fluorescence experiments are biased towards detecting T-shaped structures due to differences in oscillator strengths between those structures and parallel-stacked structures. ${ }^{17}$ Excimer formation in liquid benzene has been shown to happen quickly due to an abundance of close-to-parallel benzene pairs. ${ }^{23}$

The goal of this work is to characterize the full-dimensional nonadiabatic dynamics $^{45}$ of ultrafast excimer formation in a prototypical system for aromatic interactions, the benzene dimer in the gas phase. We started from a parallel-displaced ground-state structure since this structure shows higher stability over the T shaped types in larger stacked acene dimers. ${ }^{46,47}$ Moreover, aromatic rings in polymer structures will often exist close to parallel-oriented configurations ${ }^{3}$ due to $\pi$ - $\pi$ interactions, and excimer formation starting from these structures have been much less studied. The benzene dimer is investigated as a minimal model for aromatic stacking, and the quantitative analysis of its full-dimensional ultrafast nonadiabatic dynamics, using several transition density descriptors, allows the characterization of the excimer regarding its time evolution, density distribution, and dependence on geometric parameters. The study is carried out by promoting a $S_{0} \rightarrow S_{1}$ transition, the most direct channel for the $S_{1}$ excimer formation. The $S_{0} \rightarrow S_{1}$ channel is experimentally accessible ${ }^{14,15}$ and presents an added advantage for the simulations, since it minimizes the probability of excess vibrational energy at $S_{1}$ leading to monomer separation.

Naturally, the behavior of excimers can be quite different between aggregate and isolated systems. In a condensed phase, the mobility of monomers will be severely hindered, and even internal rotations can be impeded by other molecules. Moreover, the presence of many additional degrees of freedom allows faster redistribution of excess energy from the excitation. Nevertheless, the full-dimensional characterization of the 
basic mechanisms of excimer formation and evolution in an archetypal model like benzene dimer in the gas phase is of fundamental importance for providing vibronic information clean from noisy solvent and environmental effects.

\section{Computational details}

All electronic structure calculations were carried out using the resolution-of-the-identity (RI) algebraic diagrammatic construction to the second order $(\operatorname{ADC}(2))^{48,49}$ with the ccpVDZ basis set. ${ }^{50}$ The ADC(2) calculations were done with the TURBOMOLE 7.0 program system. ${ }^{51}$ The $\mathrm{D}_{2 \mathrm{~h}}$ symmetry was used for the parallel-ring (Figure $1 \mathrm{c}$ ) surface scans, with the benzene rings parallel to the xy-plane and two of the diametrically opposed carbon atoms located in the y-axis.

The NEWTON-X package ${ }^{52,53}$ was used for the surface-hopping dynamics ${ }^{45}$ after an $\mathrm{S}_{0} \rightarrow \mathrm{S}_{1}$ transition. 120 trajectories with time steps of $0.5 \mathrm{fs}$ were followed for $2000 \mathrm{fs}$, with the first six excited states included. Nonadiabatic events between excited states were accounted for via decoherence-corrected $(0.1 \text { Hartree })^{54}$ fewest-switches ${ }^{55}$ surface hopping, using the local diabatization approximation. ${ }^{56-60}$ Starting from the paralleldisplaced $S_{0}$ minimum of the benzene dimer in the gas phase, the initial conditions for the trajectories were sampled from a quantum-harmonic-oscillator Wigner distribution for the nuclei. ${ }^{61}$

To characterize the excitonic and charge transfer processes, the program TheoDORE ${ }^{62-66}$ was used for a transition density-matrix analysis and to generate the natural transition orbitals ${ }^{67}$ (NTOs).

Consider the transition matrix element for a transition from the ground state to the $n^{\text {th }}$ excited state in a system containing two or more fragments, expressed in a localized orbital basis:

$D_{a b}^{0 n}=\left\langle 0\left|\hat{\mathcal{E}}_{a b}\right| n\right\rangle$

Where $\hat{\varepsilon}_{a b}$ is the excitation operator and $a$ and $b$ are orbital indexes. The charge-transfer number $\Omega_{A B}^{n}$ for that excitation can be defined as:

$\Omega_{A B}^{n}=\frac{1}{2} \sum_{\mu \epsilon A} \sum_{\nu \in B}\left[\left(D^{0 n} \boldsymbol{S}\right)_{\mu \nu}\left(\boldsymbol{S} \boldsymbol{D}^{\mathbf{0 n}}\right)_{\mu \nu}+D_{\mu \nu}^{0 n}\left(\boldsymbol{S} \boldsymbol{D}^{\mathbf{0 n}} \boldsymbol{S}\right)_{\mu \nu}\right]$

Where $\mathbf{S}$ is the orbital overlap matrix and A and B are fragments of the system. This number represents the contribution of charge transfer from fragment A to fragment B (for 
$\mathrm{A} \neq \mathrm{B}$ ), and the contribution of same-fragment excitations (for $\mathrm{A}=\mathrm{B}$ ). The total $\mathrm{CT}$ character for a system with multiple fragments is given by:

$C T=\frac{1}{\Omega^{n}} \sum_{A} \sum_{B \neq A} \Omega_{A B}^{n}$

with $\Omega^{\mathrm{n}}$ standing for the total sum of the charge transfer numbers for all pairs A and B. It is also possible to estimate the average position (POS) of the excitation:

$P O S=\frac{\sum_{A} A\left(\sum_{B} \Omega_{A B}^{n}+\Omega_{B A}^{n}\right)}{2 \Omega^{n}}$

In the two-fragment case (such as the benzene dimer), POS will assume values between one and two, which are the numbers associated with each fragment. In this way, POS=1 or $\mathrm{POS}=2$ mean that the transition is completely localized in each one of the rings, while intermediary values imply a delocalized transition. The ring where the exciton starts in each trajectory was always chosen to be "fragment A". This was accomplished by substituting the POS descriptor of trajectories that start with POS $>1.5$ with an alternative POS $^{\prime}=3-$ POS, ensuring that in all cases the descriptor starts with values smaller than 1.5. To smooth out the POS curves, we have used the median of the 5 nearest data points for each point plotted in POS graphs.

\section{$3 \quad$ Results and Discussion}

\subsection{Static characterization}

We start by characterizing the excited-state surfaces along the direct ring-approach coordinates for the parallel-stacked (Figure 1c) configuration. Scans along this coordinate were calculated for six states (Figure 2), showing good agreement with the results of Krylov et al. ${ }^{18}$ The CT descriptor (Figure 3) along the scan for the first six states shows that charge transfer occurs for all four states showing energy minima ( $1{ }^{1} \mathrm{~B}_{3 \mathrm{~g}},{ }^{1}{ }^{1} \mathrm{~B}_{2 \mathrm{~g}}$ and $2{ }^{1} \mathrm{~B}_{2 \mathrm{~g}} / 2{ }^{1} \mathrm{~B}_{3 \mathrm{~g}}$ ), reaching a peak of $0.5 e$ in all of these states. This corresponds to a 50/50 mixture of local and CT character. Considering also the symmetry of the system, the excimer wavefunction can, thus, be written in the form

$\Psi=\frac{1}{2}\left(A B^{*}+A^{*} B+A^{+} B^{-}+A^{-} B^{+}\right)$ 
In other words, the excimer wavefunction is in this case an even mixture of the two local excitations $\left(A B^{*}, A^{*} B\right)$ and the two CT configurations $\left(A^{+} B^{-}, A^{-} B^{+}\right)$. It is also evident that in the first excited state (1B $3 \mathrm{~g}), \mathrm{CT}$ falls off very rapidly for distances above $2.5 \AA$. The $1{ }^{1} \mathrm{~B} 2 \mathrm{u}$ state is dissociative and does not present the same pattern, with CT always near zero. This difference between the bound and dissociative states suggests that $\mathrm{CT}$ is an essential ingredient for excimer formation in benzene.

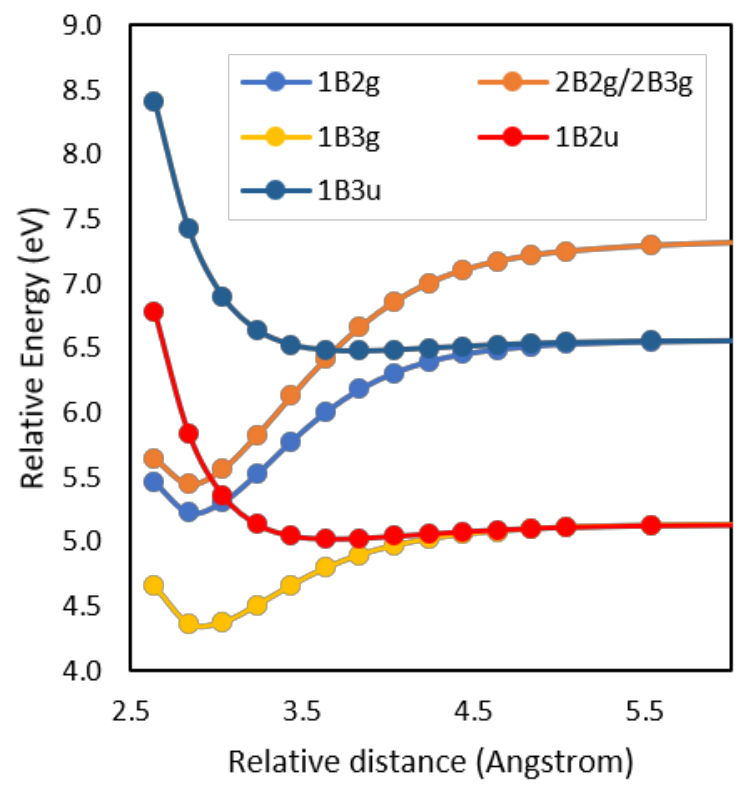

Figure 2. PES scans of the first six benzene dimer singlet excited states along the interring distance computed at the RI-ADC(2)/cc-pVDZ level. The reference energy is the ground-state energy for the rings at the largest calculated separation. 


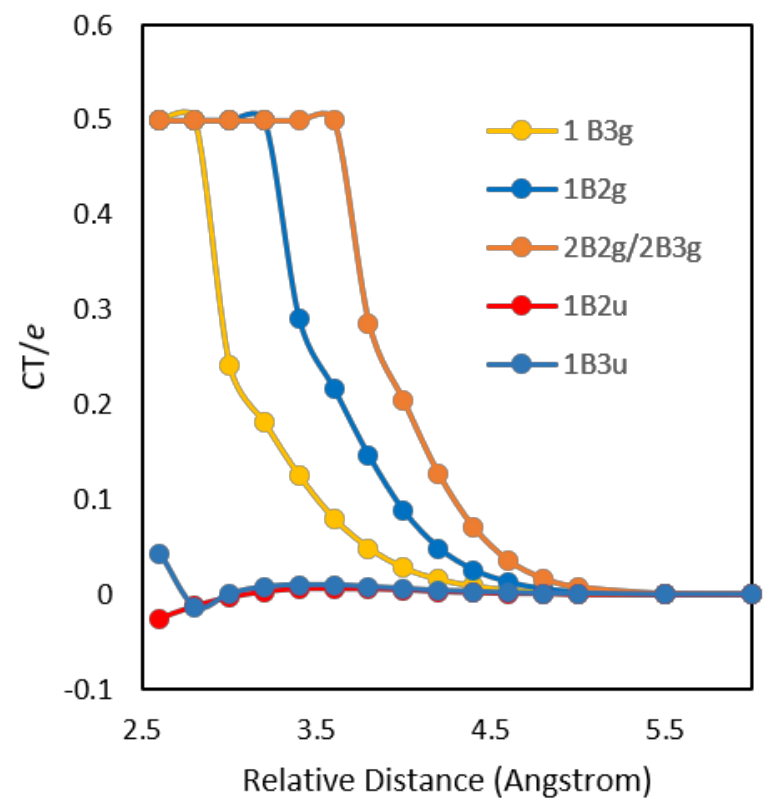

Figure 3. CT descriptor along the PES scans of the first six benzene dimer singlet excited states along the interring distance computed at the RI-ADC(2)/cc-pVDZ level.

Significant differences can be observed between the benzene-dimer excitation energies for the parallel-displaced $\mathrm{S}_{0}$ and parallel-stacked $\mathrm{S}_{1}$ minimum geometries (Table 1). In the $S_{0}$ geometry of the parallel-displaced structure, the distance between the parallel planes containing the benzene rings is $\sim 3.5 \AA$. At this geometry, the first six excited states can be thought of as three pairs of almost-degenerate $\pi-\pi^{*}$ states. This is equally true for the parallel-stacked geometry at large ring separations (>5.5 $\AA$ ). Bringing the rings closer to the parallel-stacked equilibrium geometry $(\sim 3.0 \AA)$ leads to a reduction of excitation energies for all states considered, along with a significant energy separation between two of the pairs of states. All these states present nearly zero $(<0.001)$ oscillator strength values in all considererd geometries, with the exception of the $\mathrm{S}_{3}$ state at the parallel displaced geometry, which has an oscillator strength of 0.003 (Table 1). Populating them should be strongly dependent on processes such as vibronic coupling. The $S_{1}$ transition energy at the parallel-displaced structure is $1.03 \mathrm{eV}$ larger than the $S_{1}$ transition energy at the parallel-stacked geometry. This can be taken as a rough estimation of the excess vibrational energy when the dimer is subjected to an excitation from the parallel-displaced equilibrium geometry. 
Table 1. Excitation energies $(\mathrm{eV})$ and oscillator strengths for the first six singlet excited states of the benzene dimer in the parallel-displaced and parallel-stacked (at the $S_{1}$ minimum and at the separation limit) structures.

Parallel-displaced Parallel-stacked Parallel-stacked

\begin{tabular}{ccccccc} 
& \multicolumn{2}{c}{$(3.5 \AA)$} & \multicolumn{2}{c}{$(3.0 \AA)$} & \multicolumn{2}{c}{$(5.5 \AA)$} \\
\hline \hline State & $\begin{array}{c}\text { Excitation } \\
\text { energy } \\
(\mathrm{eV})\end{array}$ & $\begin{array}{c}\text { Oscillator } \\
\text { strength }\end{array}$ & $\begin{array}{c}\text { Excitation } \\
\text { energy } \\
(\mathrm{eV})\end{array}$ & $\begin{array}{c}\text { Oscillator } \\
\text { strength }\end{array}$ & $\begin{array}{c}\text { Excitation } \\
\text { energy } \\
(\mathrm{eV})\end{array}$ & $\begin{array}{c}\text { Oscillator } \\
\text { strength }\end{array}$ \\
\hline S1 & 5.00 & 0.000 & 3.97 & 0.000 & 4.85 & 0.000 \\
S2 & 5.00 & 0.000 & 4.83 & 0.000 & 4.86 & 0.000 \\
S3 & 6.48 & 0.003 & 5.08 & 0.000 & 6.40 & 0.000 \\
S4 & 6.51 & 0.000 & 5.50 & 0.000 & 6.41 & 0.000 \\
S5 & 7.30 & 0.000 & 5.50 & 0.000 & 7.42 & 0.000 \\
S6 & 7.46 & 0.000 & 7.27 & 0.000 & 7.42 & 0.000
\end{tabular}

\subsection{Non-adiabatic Dynamics}

The dynamics starts at $S_{1}$ and mainly occurs between $S_{1}$ and $S_{2}$, without ever reaching the small $S_{0}-S_{1}$ gap regions. In very few cases, hopping to $S_{3}$ occurs, but the system never remains there for more than a few fs. Nineteen out of 120 trajectories ended prematurely due to energy convergence issues. These problems usually have to do with the system acquiring highly distorted geometries, which are not properly described at the present level of theory. Nevertheless, these trajectories offer information about the early stages of the dynamics and have not been discarded from the analysis.

The benzene rings, on average, quickly approach the parallel-stacked excimer minimum (Figure 4), but the excimer never stabilizes due to an excess of vibrational energy. This contrasts with the recently reported pyrene dimer dynamics, ${ }^{68}$ where the fragments oscillate around the minimum with decreasing amplitude of motion as vibrational energy is redistributed among the modes. 


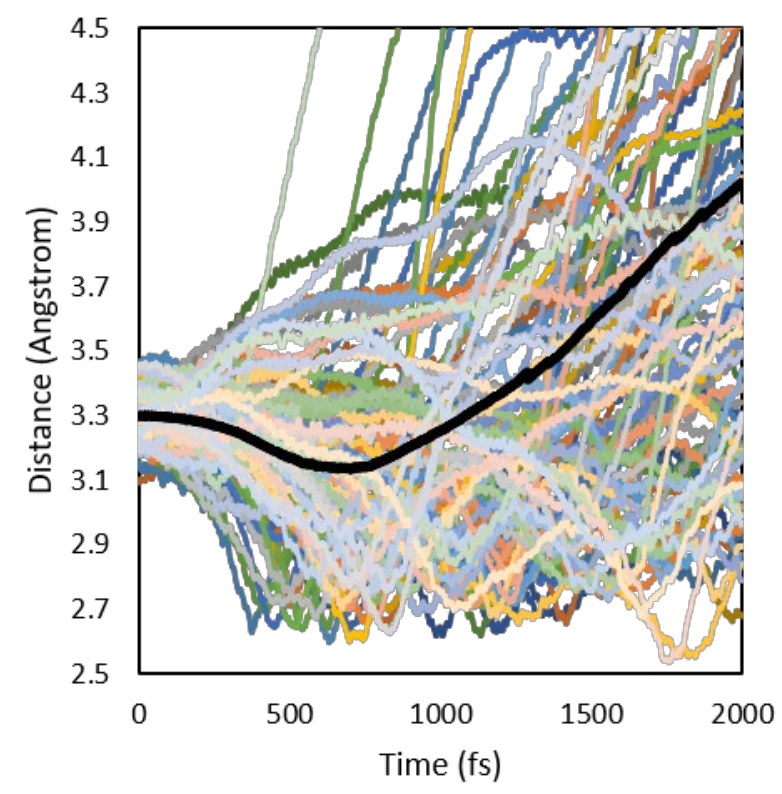

Figure 4. Distance between centers-of-mass of the two fragments along all trajectories. The black line displays the average distance taken from all trajectories.

The 2.0 ps trajectories can be separated into three types, regarding the dimer structure at the end of the dynamics: $\sim 51 \%$ of all trajectories end with the rings at a parallel or near-parallel geometry with ring distances ranging from $2.9-4.0 \AA \AA ; 40 \%$ end with the rings far apart, side-by-side or in L-shaped structures, where the rings probably interact minimally; $\sim 9 \%$ of the trajectories end in a T-shaped or skewed T-shaped dimer structure. The change from the parallel dimer to the T-shaped structure involves $S_{1}-S_{2}$ gap oscillations akin to those that appear in the parallel dimer formation, which are discussed later in Figure 10. The $\mathrm{CT}$ index remains below 0.03 during ring rotation in all such trajectories, indicating that the charge-transfer character is negligible. The $\mathrm{S}_{1}$ and $\mathrm{S}_{2}$ adiabatic populations of the ensemble oscillate considerably in the first $450 \mathrm{fs}$, while the system leaves the Franck-Condon region, and then stabilizes at around $0.87\left(\mathrm{~S}_{1}\right)$ and 0.13 $\left(\mathrm{S}_{2}\right)$. As the dynamics approaches its end, the populations appear to slowly grow farther apart, but larger simulation times would be needed to confirm this trend (Figure 5). 


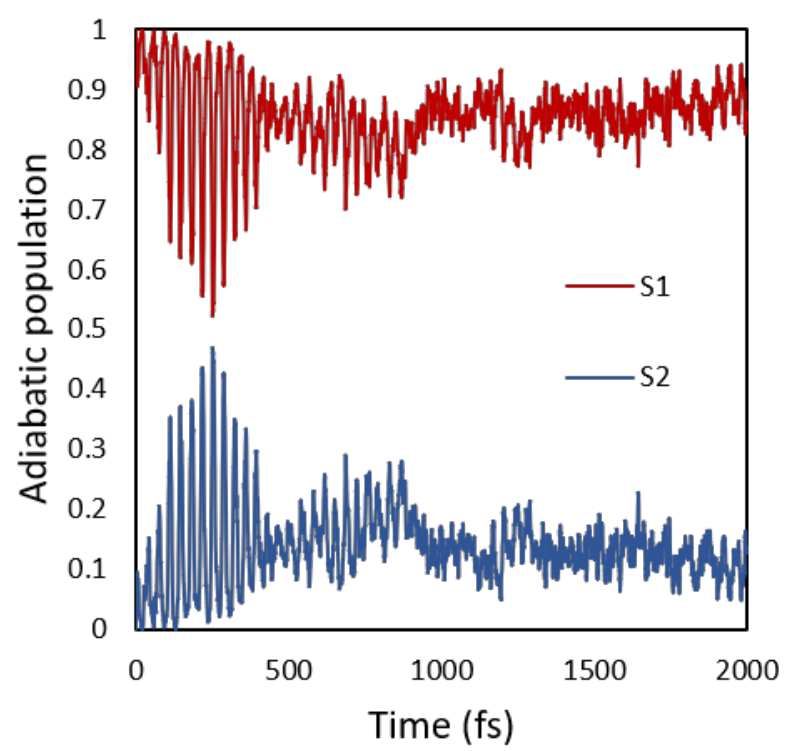

Figure 5. Adiabatic populations of the $S_{1}$ and $S_{2}$ states for the ensemble of trajectories.

The information of CT variation along the trajectories and its correlation with the proximity of the ring structures to the parallel-stacked minimum, which is associated with the excimer, is an important one, albeit not so straightforward to present in a compact way. To do this, we established that the rings should be considered "near" (as in "near the excimer region") when their centers of mass are at a distance smaller or equal to the distance at the minimum in the $S_{1}$ PES (3.0 $\AA$ ) and "far" otherwise. This choice has been adopted to count fewer structures where the centers of mass are close, but the rings are not close to a parallel-stacked geometry. The CT values were averaged for all trajectories, along their whole duration, and compared with a similar separate average for "near" and "far" timespans. The maximum CT values for each "near" and "far" timespan were also collected, and then averaged over all trajectories. These data are presented in Table 2.

Table 2. Average CT and average maximum CT for the benzene dimer in configurations near $(<3.0 \AA)$ and far $(>3.0 \AA)$ to the excimer minimum structure based on center-of-mass (c.o.m.) distance.

c.o.m.
distance $(\AA)$$\quad$ Avg. CT (e) Avg. $\mathrm{CT}_{\max }(e)$




\begin{tabular}{lll}
\hline$<3.0$ & 0.10 & 0.20 \\
$>3.0$ & 0.01 & 0.07 \\
\hline
\end{tabular}

Average CT increases 10-fold for configurations where the rings are near each other in comparison to configurations with the rings far from each other. The average maximum CT attained at "near" configurations is approximately three times larger than at "far" configurations, evidencing the relevance of partial charge transfer character in excimer formation in the $S_{1}$ state. Figure 6 further illustrates this relationship by showing the center of mass and the CT descriptor along a representative trajectory. Again, increases in CT correlate with the rings close approach, further implying the importance of some degree of charge-transfer character for the benzene excimer.

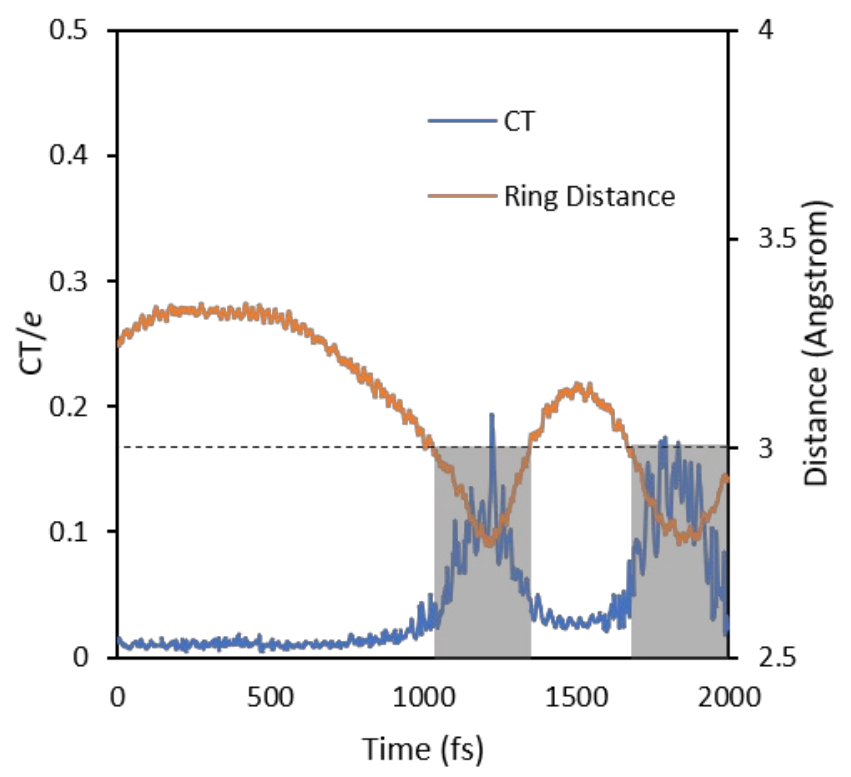

Figure 6. Correlation between benzene ring approach in the excited state and increase in CT character for one representative trajectory. Shaded areas correspond to timeframes where the fragments are at $3.0 \AA$ distance or less.

The identification of the ring approach along the trajectories in this manner also allows the estimation of times scales regarding the dynamics of excimer formation. More than $67 \%$ of the visits to the "near" region start before the 1 ps mark, most of them between 0.5 and $1.0 \mathrm{ps}$, and as the dynamics evolves, the region is less visited (Figure 7); this is a small fraction of the 18 ps obtained by Miyazaki and Fujii ${ }^{21}$ in gas-phase experiments where the T-shaped dimer structures are assumed to be dominant in $S_{0}$. It is 
reasonable to conclude that this large difference is attributable to the time it takes for the benzene rings to rearrange from the T-shaped structure towards the parallel structure.

Approximately $80 \%$ of each of these visits lasts no more than 400 fs (Figure 8); this rather small time is probably related to excess vibrational energy from the $S_{0} \rightarrow S_{1}$ excitation. This means that an isolated dimer excited from a parallel-displaced geometry might not stay in the excimer potential well long enough for it to be detected, though this does not exclude its formation by this process in aggregated systems.

The use of the Wigner distribution for obtaining initial conditions for classical nuclear dynamics simulations can lead to zero-point energy (ZPE) leakage from higher frequency vibrational modes to lower frequency ones ${ }^{69-72}$. Since most of the kinetic energy is concentrated in the high-frequency modes of the hydrogen atoms, it is possible to get an estimate on how impactful the leakage is by following the ratio between the kinetic energy of hydrogen atoms at time $t$ and the kinetic energy of hydrogen atoms at time zero, both computed in the excited state and averaged over all trajectories. Figure S1 (Supporting Information) shows the evolution of this average ratio. From this figure, one can see that the $\mathrm{H}$-atom kinetic energy reduces about $15 \%$ during the dynamics, which seems to indicate some ZPE leakage. This overall analysis, however, does not account for the distribution of the leaked kinetic energy over the different modes. Due to the larger vibrational couplings, intramolecular modes should absorb most of this energy overflow. Therefore, our excimer dynamics, which mostly depends on the weakly-coupled intermolecular modes, should be only slightly affected. 


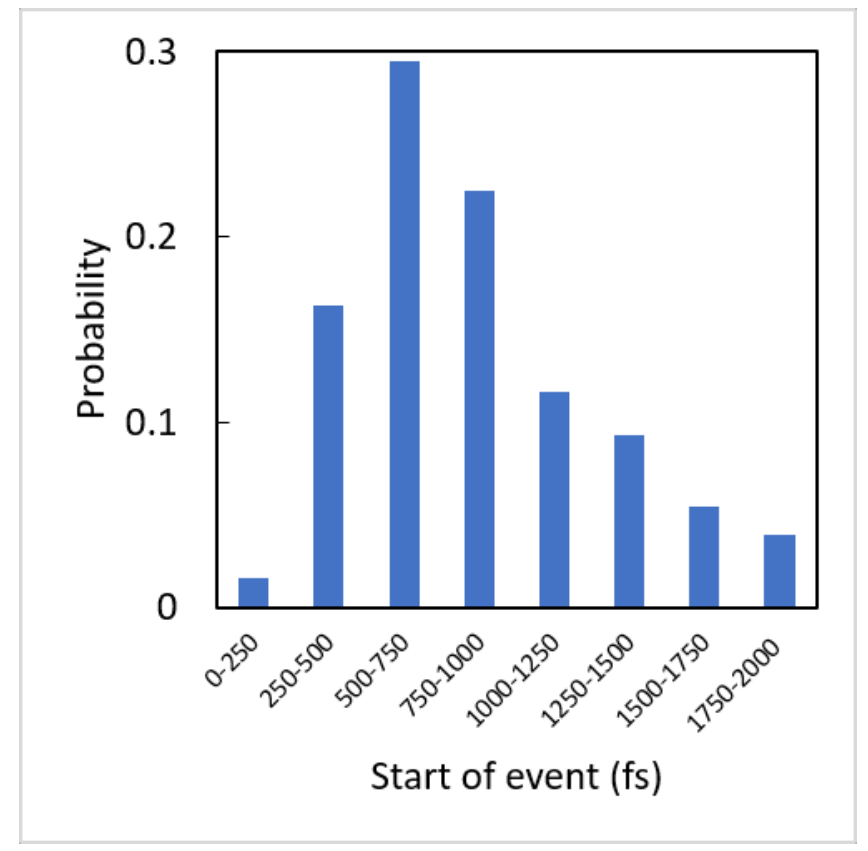

Figure 7. Fraction of trajectories where fragments approach the "near" region (distances smaller than $3.0 \AA$ ) in each 250 fs time slice, obtained by counting the number of times such an approach starts at each time slice in all trajectories. Re-entering the "near" region is counted as an independent event.

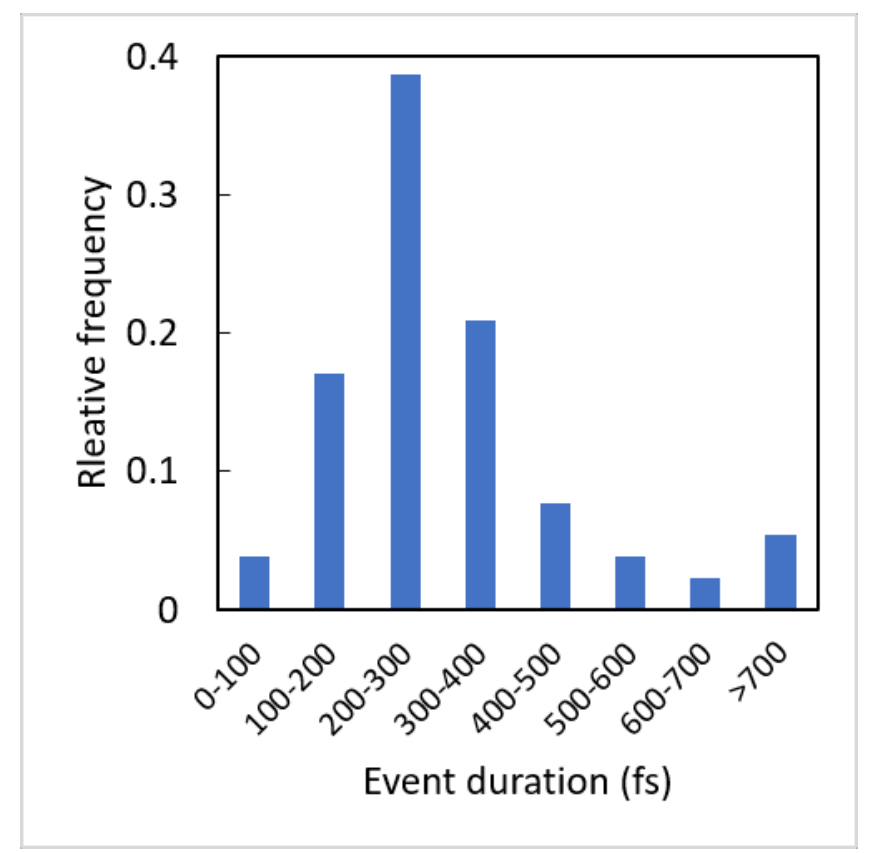

Figure 8. Frequency of the different durations of fragments in the "near" regions in all trajectories. 
The localization of the exciton during the dynamics was accompanied by following the POS descriptor along the trajectories. As described in the Computational Details, the benzene ring where the exciton is initially localized is taken conventionally as fragment $\mathrm{A}$. The frequencies with which the exciton was primarily localized in A (1.0 $\leq \mathrm{POS}<1.33)$, in $\mathrm{B}(1.66<\mathrm{POS} \leq 2.00)$ or delocalized between the two rings $(1.33<$ POS $<1.66$ ) are collected in time slices of $250 \mathrm{fs}$ in Figure 9. As the dynamics evolves, the exciton tends to distribute between the two rings with approximately the same probability, around $45 \%$. The probability of finding a delocalized exciton at 2 ps is less than $10 \%$. The results suggest that the incoherent exciton evolution, here simulated by independent surface hopping trajectories, quickly erases the dynamics memory, and at around $1 \mathrm{ps}$ it is not possible to pinpoint the original exciton source anymore.

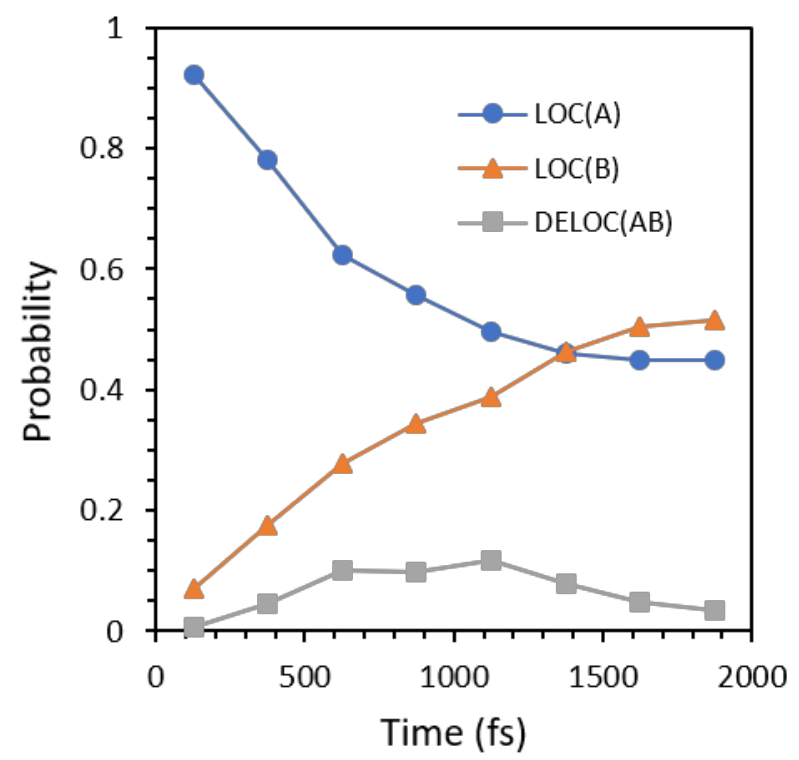

Figure 9. Probability of finding localized and delocalized excitons as a function of time.

The analysis of a representative trajectory displaying many of the typical features of the benzene dimer excited state dynamics is summarized in Figure 10. The $S_{1}-S_{2}$ gap oscillates considerably during the trajectories, typically going from zero or near zero to to $1.1 \mathrm{eV}$ (e.g., the 240-570 fs range in Figure 10A). These fast oscillations ( $\sim 25-70$ fs for a full period) are associated with structural motion unrelated to the ring approach, such as intra-ring mode vibrations, and quick visits to the $S_{2}$ state occurring near the zero-gap 
region. In most cases, when the rings get close to the excimer minimum $(<3.0 \AA$ ring distance), the lower limit of the $S_{1}-S_{2}$ gap increases which corresponds to a gap widening (Figure 10A). This agrees with the $S_{1}-S_{2}$ separation increase characterized in Figure 2.

There are two pairs of NTOs characterizing each excited state, which we shall refer to as $\mathrm{NTO}_{\mathrm{a}} \rightarrow \mathrm{NTO}_{\mathrm{a}}{ }^{*}$ and $\mathrm{NTO}_{\mathrm{b}} \rightarrow \mathrm{NTO}_{\mathrm{b}}{ }^{*}$. When the rings are far from the excimer minimum the $S_{1}$ and $S_{2}$ excited states can be characterized by two $\pi \rightarrow \pi^{*}$ orbital excitations localized in each ring, respectively (Figure 11). In these cases, when the $S_{1}-S_{2}$ gap is large, the system occupies the $S_{1}$ state; the hops to the $S_{2}$ state that occur near the $S_{1}-S_{2}$ zero-gap regions correspond to a mere switch of the rings where the $S_{1}$ and $S_{2}$ NTOs are located, typical of diabatic trapping, i.e. the diabatic state character stays the same while the adiabatic state switches. ${ }^{73}$

Exciton position POS can change during the hops, but they do not necessarily correlate with lasting changes in the adiabatic populations (Figure 10B and 10C). In the example, as the benzene rings approach each other, a sudden change in POS happens at around $500 \mathrm{fs}$, which is accompanied by a quick inversion of adiabatic populations where the $S_{1}$ and $S_{2}$ states are near-degenerate. As the molecules move even closer, the $S_{1}-S_{2}$ gap widens and the adiabatic population is once again fixed at $S_{1}$, while the POS deviates from its strictly localized behavior. The largest oscillations in the exciton position will occur during ring approach, and charge transfer character typically increases in visits to the excimer region (Figure 10D). This is further evidenced by the delocalization of the NTOs across the two benzene fragments at the excimer structure (Figure 12). The redistribution of the excitons among the benzene rings described in Figure 8 is connected to the ring approach by this electronic process. 


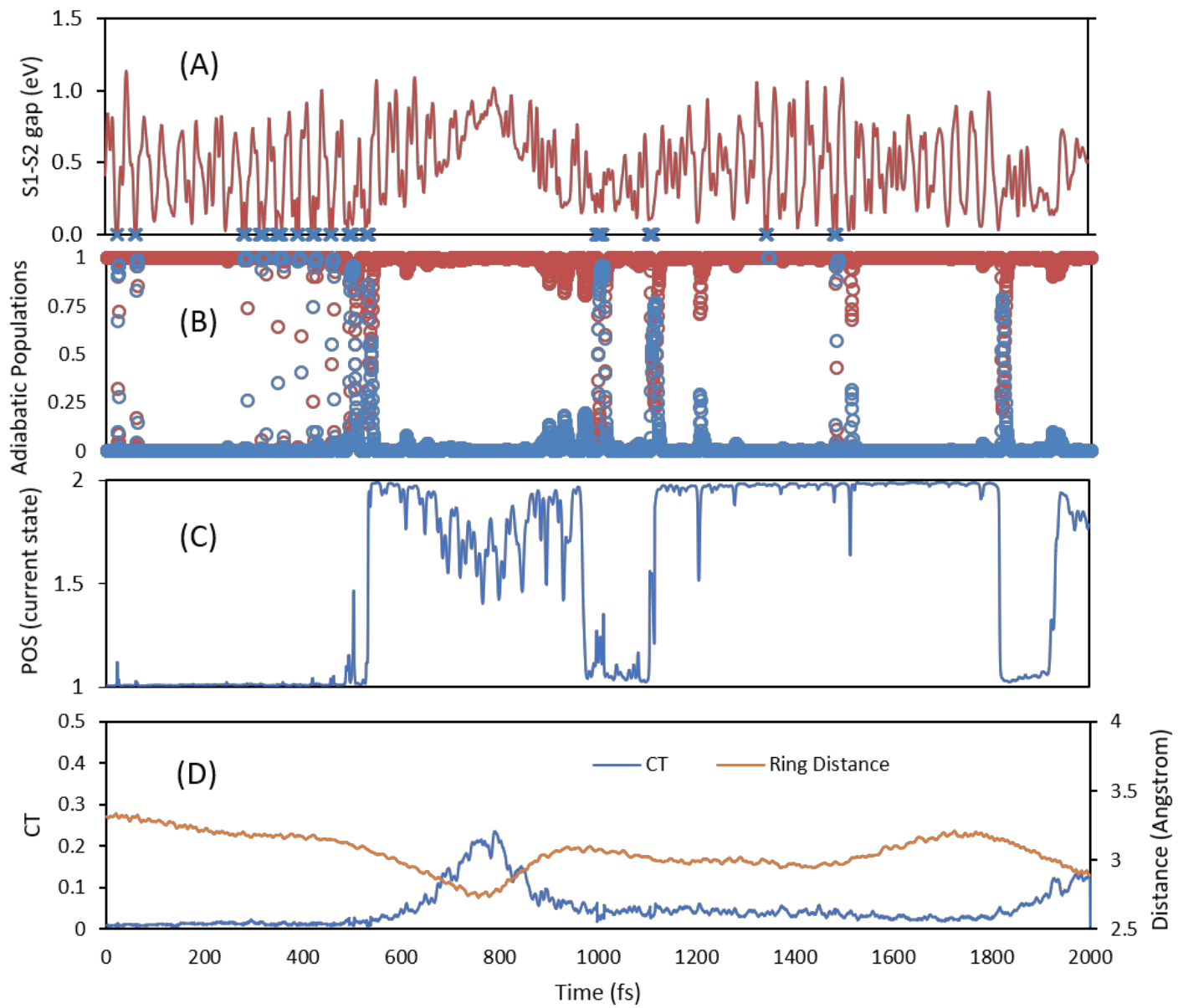

Figure 10. Anatomy of a representative trajectory. A) $S_{1}-S_{2}$ gap. The blue crosses mark points where the dynamics is found in the $\mathrm{S}_{2}$ state. B) Adiabatic populations. Red circles correspond to population of the first excited state and blue circles to second excited state populations. C) Exciton localization (POS) along the trajectory. D) CT and fragment (center-of-mass) distance, along the trajectory. 


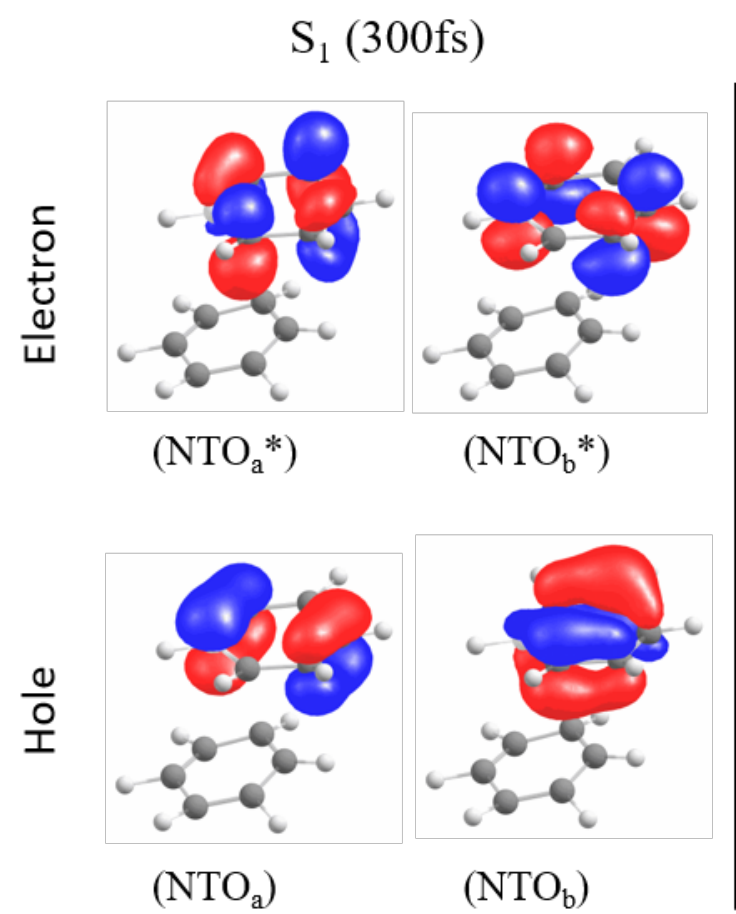

Occ. $\quad 0.38$

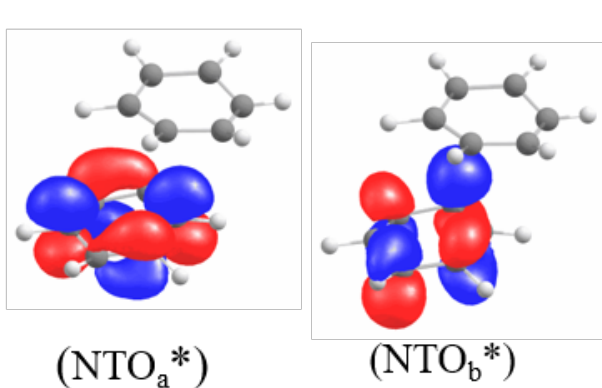

$\left(\mathrm{NTO}_{\mathrm{a}}^{*}\right)$

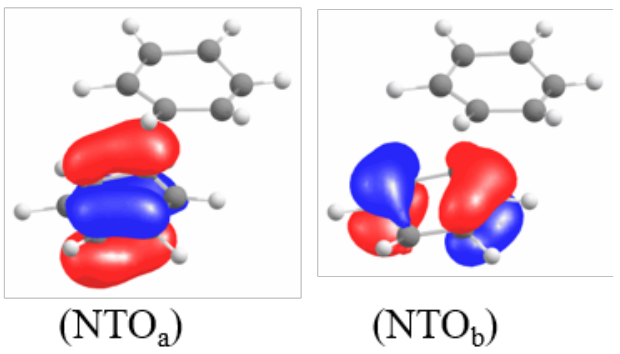

0.37

0.57

Figure 11. Natural transition orbitals for the $S_{1}$ and $S_{2}$ states for rings far from the excimer minimum. $S_{1}$ is the occupied state. The hole NTOs are at the bottom and the electron NTOs are at the top. The NTO electron-hole occupations pair are printed below each pair. 

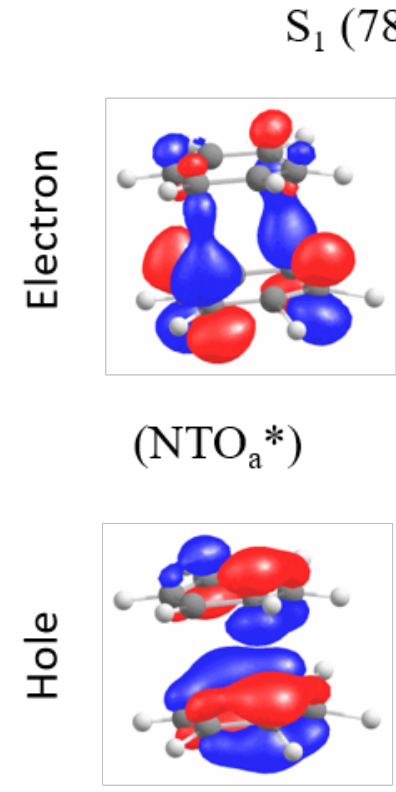

$\left(\mathrm{NTO}_{\mathrm{a}}\right)$ (783.5fs)
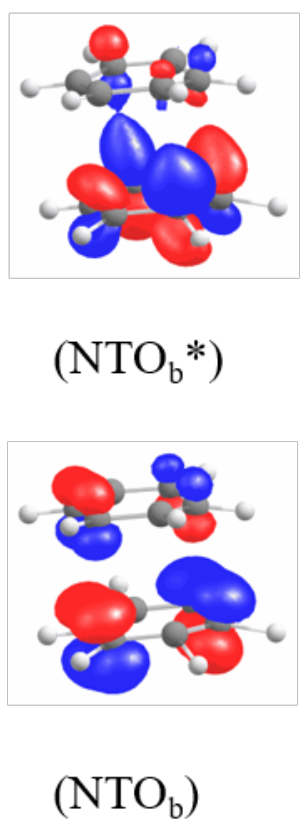

0.51
$\mathrm{S}_{2}(783.5 \mathrm{fs})$
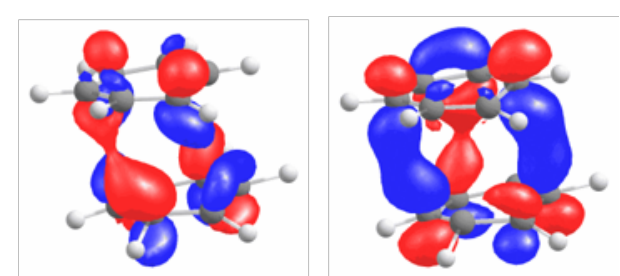

$\left(\mathrm{NTO}_{\mathrm{a}}^{*}\right)$

$\left(\mathrm{NTO}_{\mathrm{b}} *\right)$

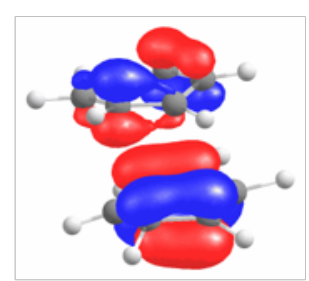

$\left(\mathrm{NTO}_{a}\right)$

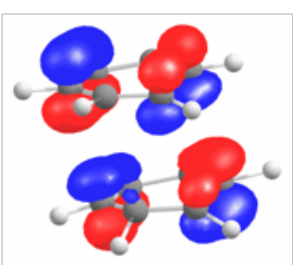

$\left(\mathrm{NTO}_{\mathrm{b}}\right)$

Occ. $\quad 0.41$

Figure 12. Natural transition orbitals for the $S_{1}$ and $S_{2}$ states near the excimer minimum. $S_{1}$ is the occupied state.

It is instructive to contrast these results to those from the recent paper on gas-phase pyrene excimer formation. ${ }^{68}$ The $S_{1}-S_{2}$ gap widening at excimer formation observed in the benzene dimer dynamics also occurs in the pyrene dimer case, as do the fast $S_{1}-S_{2}$ switching. The estimated time for pyrene approach in ref. ${ }^{68}$ is $100 \mathrm{fs}$, a five to tenfold reduction in comparison to our estimates for the benzene excimer. The damped oscillations around the excimer potential well, which precede the stabilization of the excimer, are estimated to be $\sim 350$ fs for the pyrene dimer, which is similar to the 200-400 fs duration of visits to the excimer region in the benzene case. The small timeframes for ring approach are probably general for arenes starting from parallel or near-parallel structures and the determining factor for the timescales of excimer formation in these systems should be the redistribution of excess vibrational energy. 


\section{Conclusions}

The dynamics of excimer formation from parallel-displaced structures of an isolated benzene dimer can be summarized in the following way: the excess energy from the hot transition to $S_{1}$ leads to a fast approach of the benzene rings towards the excimer parallelstacked structure ( 0.5-1.0 ps), with ring distances reaching $3.0 \AA$ and less. The available excess vibrational energy of $\sim 1.0 \mathrm{eV}$ is responsible for the fact that the two molecules do not remain near the excimer potential well for long, taking, on average, around 0.25-0.3 ps to leave this region. These visits to the excimer structure are short but sufficient to promote a change in the CT character of the states (about $20 \%$ on average) and also quickly lead to an even redistribution of the exciton localization between the two rings. The information about the initially excited monomer is lost after 1 ps. The approach of the monomers is observed to reoccur in some cases, meaning that it is possible for the dimer to remain near the parallel-stacked geometry for longer times. However, with few possibilities to lose the excess excitation energy, the isolated dimer cannot form a stable excimer from the parallel-displaced structure and should not be easily detectable by traditional fluorescence experiments. In extended systems or in the condensed phase, the hindered motion of groups, together with more pathways for vibrational energy redistribution allows the formation of the excimer. ${ }^{23}$ The typically reported timescales for excimer formation in benzene (tens of ps) are mostly due to vibrational energy redistribution and exceed by at least an order of magnitude the timescale needed for exciton hopping between benzene rings. This means that there is a time window after excitation when excitons should be able to diffuse before they are trapped by excimer formation.

\section{Acknowledments}

TMC and IB thank the Brazilian Agency CAPES for support of this work in the framework of the Science without Borders Brazilian Program. MB thanks the support of the Excellence Initiative of Aix-Marseille University (A*MIDEX) and the project Equip@Meso (ANR-10-EQPX-29-01), both funded by the French Government "Investissements d'Avenir" program. MB also acknowledges funding from the WSPLIT project (ANR-17-CE05-0005-01). This work was supported by the Center for Integrated Nanotechnologies (Project No. C2013A0070), an Office of Science User Facility 
operated for the U.S. Department of Energy Office of Science by Los Alamos National Laboratory (Contract DE-AC52-06NA25396) and Sandia National Laboratories (Contract DE-AC04-94AL85000). Computer time at the computer cluster Arran of the School of Pharmaceutical Science and Technology, Tianjin University, Tianjin, China and the Vienna Scientific Cluster (Project nos. 70376), Vienna, Austria, is gratefully acknowledged.

\section{References}

1. B. Stevens and E. Hutton, Nature, 1960, 186, 1045.

2. J. B. Birks, Rev Prog Phys, 1975, 38, 903.

3. J. Mei, N. L. Leung, R. T. Kwok, J. W. Lam and B. Z. Tang, Chem Rev, 2015, $115,11718-11940$.

4. X. Feng, A. V. Luzanov and A. I. Krylov, The Journal of Physical Chemistry Letters, 2013, 4, 3845-3852.

5. C. E. Miller, M. R. Wasielewski and G. C. Schatz, The Journal of Physical Chemistry C, 2017, 121, 10345-10350.

6. J. N. Schrauben, J. L. Ryerson, J. Michl and J. C. Johnson, J Am Chem Soc, 2014, $136,7363-7373$.

7. H. L. Stern, A. J. Musser, S. Gelinas, P. Parkinson, L. M. Herz, M. J. Bruzek, J. Anthony, R. H. Friend and B. J. Walker, Proc Natl Acad Sci U S A, 2015, 112, 7656-7661.

8. C. E. Crespo-Hérnandez, B. Cohen, P. M. Hare and B. Kohler, Chem Rev, 2004, $104,1977-2019$.

9. F. Plasser, A. J. Aquino, H. Lischka and D. Nachtigallova, Top Curr Chem, 2015, $356,1-37$.

10. O. G. Reid, R. D. Pensack, Y. Song, G. D. Scholes and G. Rumbles, Chemistry of Materials, 2014, 26, 561-575.

11. A. Nowak-Krol, B. Fimmel, M. Son, D. Kim and F. Wurthner, Faraday Discuss, $2015,185,507-527$.

12. P. E. Hartnett, A. Timalsina, H. S. Matte, N. Zhou, X. Guo, W. Zhao, A. Facchetti, R. P. Chang, M. C. Hersam, M. R. Wasielewski and T. J. Marks, J Am Chem Soc, 2014, 136, 16345-16356.

13. M. T. Vala, I. H. Hillier, S. A. Rice and J. Jortner, J Chem Phys, 1966, 44, 23-35. 
14. T. Hirata, H. Ikeda and H. Saigusa, J Phys Chem A, 1999, 103, 1014-1024.

15. H. Saigusa, M. Morohoshi and S. Tsuchiya, J Phys Chem A, 2001, 105, 7334.

16. J. C. Amicangelo, J Phys Chem A, 2005, 109, 9174-9182.

17. F. A. Balmer, M. A. Trachsel, A. van der Avoird and S. Leutwyler, J Chem Phys, $2015,142,234306$.

18. K. Diri and A. I. Krylov, J Phys Chem A, 2012, 116, 653-662.

19. M. Kolaski, C. R. Arunkumar and K. S. Kim, J Chem Theory Comput, 2013, 9, $847-856$.

20. M. Miyazaki and M. Fujii, Phys Chem Chem Phys, 2017, 19, 22759-22776.

21. M. Miyazaki and M. Fujii, Phys Chem Chem Phys, 2015, 17, 25989-25997.

22. H. Shinohara and N. Nishi, J Chem Phys, 1989, 91, 6743-6751.

23. E. S. Iyer, A. Sadybekov, O. Lioubashevski, A. I. Krylov and S. Ruhman, J Phys Chem A, 2017, 121, 1962-1975.

24. K. O. Börnsen, H. L. Selzle and E. W. Schlag, J Chem Phys, 1986, 85, 1726-1732.

25. P. Hobza, H. L. Selzle and E. W. Schlag, J Chem Phys, 1993, 97, 3937-3938.

26. M. O. Sinnokrot, E. F. Valeev and C. D. Sherrill, J Am Chem Soc, 2002, 124, 10887-10893.

27. M. O. Sinnokrot and C. D. Sherrill, J Phys Chem A, 2004, 108, 10200-10207.

28. T. Janowski and P. Pulay, Chem Phys Lett, 2007, 447, 27-32.

29. E. Arunan and H. S. Gutowsky, J Chem Phys, 1993, 98, 4294-4296.

30. T. Ebata, M. Hamakado, S. Moriyama, Y. Morioka and M. Ito, Chem Phys Lett, 1992, 199, 33-41.

31. P. M. Felker, P. M. Maxton and M. W. Schaeffer, Chem Rev, 1994, 94, 17871805.

32. B. F. Henson, G. V. Hartland, V. A. Venturo and P. M. Felker, J Chem Phys, 1992, 97, 2189-2208.

33. V. A. Venturo and P. M. Felker, J Chem Phys, 1993, 99, 748-751.

34. R. Podeszwa, R. Bukowski and K. Szalewicz, J Phys Chem A, 2006, 110, 1034510354

35. A. van der Avoird, R. Podeswa, K. Szalewicz, C. Leforestier, R. van Harrevelt, P.

R. Bunker, M. Schnell, G. von Helden and G. Meijer, Phys Chem Chem Phys, 2010, 12, 8219-8240.

36. M. Pitonak, P. Neogrády, J. Rezác, P. Jurecka, M. Urban and P. Hobza, J Chem Theory Comput, 2008, 4, 1829-1834. 
37. M. S. Marshall, L. A. Burns and C. D. Sherrill, J Chem Phys, 2011, 135, 194102.

38. M. S. Marshall and C. D. Sherrill, J Chem Theory Comput, 2011, 7, 3978-3982.

39. P. M. Gill and H. J. Werner, Phys Chem Chem Phys, 2008, 10, 3318-3319.

40. R. Podeszwa, K. Patkowski and K. Szalewicz, Phys Chem Chem Phys, 2010, 12, 5974-5979.

41. G. Knizia, T. B. Adler and H. J. Werner, J Chem Phys, 2009, 130, 054104.

42. J. B. Hopkins, D. E. Powers and R. E. Smalley, J Phys Chem, 1981, 85, 37393742.

43. P. R. R. Langridge-Smith, D. V. Brumbaugh, C. A. Haynam and D. H. Levy, $J$ Phys Chem, 1981, 85, 3742-3746.

44. W. Radloff, T. Freudenberg, H.-H. Ritze, V. Stert, F. Noack and I. V. Hertel, Chem Phys Lett, 1996, 261, 301-306.

45. R. Crespo-Otero and M. Barbatti, Chem. Rev., 2018, DOI:10.1021/acs.chemrev.1027b00577.

46. A. Reyes, M. A. Tlenkopatchev, L. Fomina, P. Guadarrama and S. Fomine, J Phys Chem A, 2003, 107, 7027-7031.

47. C. Gonzalez and E. C. Lim, J Phys Chem A, 2000, 104, 2953-2957.

48. A. B. Trofimov and J. Schirmer, J. Phys. B: At., Mol. Opt. Phys., 1995, 28, 22992324.

49. A. Dreuw and M. Wormit, WIREs: Comp. Mol. Sci., 2015, 5, 82-95.

50. T. H. Dunning Jr, J. Chem. Phys., 1989, 90, 1007-1023.

51. R. Ahlrichs, M. Bär, M. Häser, H. Horn and C. Kölmel, Chem. Phys. Lett., 1989, $162,165-169$.

52. M. Barbatti, M. Ruckenbauer, F. Plasser, J. Pittner, G. Granucci, M. Persico and H. Lischka, WIREs: Comp. Mol. Sci., 2014, 4, 26-33.

53. M. Barbatti, G. Granucci, M. Ruckenbauer, F. Plasser, R. Crespo-Otero, J. Pittner, M. Persico and H. Lischka, 2017.

54. G. Granucci and M. Persico, J. Chem. Phys., 2007, 126, 134114-134111.

55. J. C. Tully, J. Chem. Phys., 1990, 93, 1061-1071.

56. F. Plasser, G. Granucci, J. Pittner, M. Barbatti, M. Persico and H. Lischka, J. Chem. Phys., 2012, 137, 22A514-513.

57. G. Granucci, M. Persico and A. Toniolo, J. Chem. Phys., 2001, 114, 10608-10615.

58. S. Hammes-Schiffer and J. C. Tully, Journal of Chemical Physics, 1994, 101, 4657-4667. 
59. F. Plasser, R. Crespo-Otero, M. Pederzoli, J. Pittner, H. Lischka and M. Barbatti, Journal of Chemical Theory and Computation, 2014, 10, 1395-1405.

60. M. Barbatti, J. Pittner, M. Pederzoli, U. Werner, R. Mitrić, V. Bonačić-Koutecký and H. Lischka, Chemical Physics, 2010, 375, 26-34.

61. R. Crespo-Otero and M. Barbatti, Theor. Chem. Acc., 2012, 131, 1237.

62. F. Plasser and H. Lischka, J Chem Theory Comput, 2012, 8, 2777-2789.

63. F. Plasser, M. Wormit and A. Dreuw, J Chem Phys, 2014, 141, 024106.

64. F. Plasser, S. A. Bappler, M. Wormit and A. Dreuw, J Chem Phys, 2014, 141, 024107.

65. F. Plasser, B. Thomitzni, S. A. Bappler, J. Wenzel, D. R. Rehn, M. Wormit and A. Dreuw, J Comput Chem, 2015, 36, 1609-1620.

66. F. Plasser, "TheoDORE 1.2.3: a package for theoretical density, orbital relaxation, and exciton analysis" available from http://theorodore-qc.sourceforge.net

67. R. L. Martin, J Chem Phys, 2003, 118, 4775-4777.

68. J. Hoche, H. C. Schmitt, A. Humeniuk, I. Fischer, R. Mitric and M. I. S. Rohr, Phys Chem Chem Phys, 2017, 19, 25002-25015.

69. S. Habershon and D. E. Manolopoulos, J Chem Phys, 2009, 131, 244518.

70. M. Persico and G. Granucci, Theor Chem Acc, 2014, 133, 1526.

71. F. Brieuc, Y. Bronstein, H. Dammak, P. Depondt, F. Finocchi and M. Hayoun, $J$ Chem Theory Comput, 2016, 12, 5688-5697.

72. J. Suchan, D. Hollas, B. F. E. Curchod and P. Slavicek, Faraday Discuss, 2018, DOI: $10.1039 / \mathrm{c} 8 \mathrm{fd} 00088 \mathrm{c}$.

73. F. Plasser and H. Lischka, J Chem Phys, 2011, 134, 034309. 